\title{
Retroperitoneal Course of Iliohypogastric, Ilioinguinal, and Genitofemoral Nerves: A Study to Improve Identification and Excision During Triple Neurectomy
}

\author{
NDI GEH, ${ }^{1}$ MIKE SCHULTZ, ${ }^{1}$ LYNDA YANG, $^{2}$ AND JOHN ZELLER $3,4,5 *$ \\ ${ }^{1}$ University of Michigan Medical School, Ann Arbor, Michigan \\ ${ }^{2}$ Department of Neurosurgery, University of Michigan Medical School, Ann Arbor, Michigan \\ ${ }^{3}$ Department of Surgery, University of Michigan Medical School, Ann Arbor, Michigan \\ ${ }^{4}$ Department of Orthopedic Surgery, University of Michigan Medical School, Ann Arbor, Michigan \\ ${ }^{5}$ Department of Emergency Medicine, University of Michigan Medical School, Ann Arbor, Michigan
}

Triple neurectomy of the iliohypogastric (IHN), ilioinguinal (IIN), and genitofemoral (GFN) nerves is an available treatment option for chronic groin pain when conservative measures are ineffective. This research study attempted to define the variability of IHN, IIN, and GFN by categorizing variation and establishing a relationship to clinically significant landmarks. 22 cadavers (43 specimens) were dissected. Age, gender, ethnicity, BMI, and pertinent medical history were recorded for each specimen. Nerve emergence, insertion, and split points were measured in relation to clinically significant landmarks. Retroperitoneal trajectories of IHN, IIN, and GFN were analyzed and categorized based on nerve branching patterns. IIN and IHN had three branching patterns - type A (47\%) in which the IIH and IIN exit as separate branches; type B (26\%) in which the IIH and IIN exit as a single bundle and split; and type C $(28 \%)$ in which the IIH and IIN exit and do not split. The GFN had three branching patterns-type $1(50 \%)$ in which the GFN exited from the psoas major and then split into the genital and femoral branches; type $2(30 \%)$ in which the GFN exited and did not split; and type $3(20 \%)$ in which the GFN exited the psoas major already split into the genital and femoral branches. Variations in the IHN, IIN, and GFN nerves outlined in this study will provide surgeons with clinically useful information aiding in successful and efficient localization of these nerves during retroperitoneal procedures, including laparoscopic triple neurectomy. Clin. Anat. 28:903-909, 2015. ๑ 2015 Wiley Periodicals, Inc.

Key words: iliohypogastric; ilioinguinal; genitofemoral; triple neurectomy; chronic groin pain

\footnotetext{
Abbreviations used: FB, femoral branch; GB, genital branch; GFN, genitofemoral; IHN, iliohypogastric; IIN, ilioinguinal; PDJ, psoas-diaphragm junction; TA, transversus abdominis.

Contract grant sponsor: University of Michigan Medical School's SBR Program.

*Correspondence to: John L. Zeller, MD, PhD, 1137 East Catherine St, Room 3767E, Med Sci Building II, Ann Arbor, Michigan 48109. E-mail: jlzeller@umich.edu
}

Received 17 May 2015; Revised 26 June 2015; Accepted 26 June 2015

Published online 16 July 2015 in Wiley Online Library (wileyonlinelibrary.com). DOI: 10.1002/ca.22592 


\section{INTRODUCTION}

The incidence of chronic groin pain following hernia repair has been documented to range from $12 \%$ to $43 \%$ (Cunningham et al., 1996; Courtney et al., 2002). Surgical management via neurectomy of the iliohypogastric (IHN), ilioinguinal (IIN), and genitofemoral (GFN) nerves is currently an available treatment option for chronic groin pain when conservative measures have failed (Tender and Serban, 2013). Traditionally, this procedure was completed as an open, 2-stage approach involving an anterior incision of the inguinal region to reach the IHN and IIN and a flank incision to reach the GFN (Amid, 2004). Recently, a 1-stage laparoscopic triple neurectomy has been described (Krahenbuhl et al., 1997; Muto et al., 2005; Giger et al., 2009; Song et al., 2011). Song and colleagues documented their initial three experiences with this procedure. Two patients with chronic groin pain exhibited significant relief at 10 months and 3 years post operatively, following transection of the three nerves. A third patient, after undergoing this procedure, experienced recurrent pain along the medial aspect of his groin two months post operatively. Clinical examination suggested that only the femoral branch (FB) of the GFN had been cut. This patient required an open reexploration through a flank incision to transect the genital portion of the GFN. As the utility of laparoscopic triple neurectomy increases, it is necessary to gain a better understanding of nerve variation within the retroperitoneum. Inadequate pain relief after triple neurectomy was reported in $26 \%$ of patients with neuropathic groin pain due to GFN entrapment with or without IIN entrapment (Giger et al., 2009).

Classical descriptions of anatomy identify the GFN piercing the psoas major and emerging on the anterior surface of the muscle. It descends distally and divides anterior to the distal third of the psoas into its femoral and genital branches (GBs). The IHN courses posterior to the psoas major and exits through its lateral border anterior to the quadratus lumborum and iliacus muscles. Near the iliac crest, it pierces the transversus abdominis (TA). The IIN follows a similar pattern (Arslan, 2005). While this description and others like it, (Clemente, 1997; Moore and Dalley, 2006; Netter, 2006) may serve as a rule of thumb, they do not take into account the variation in these nerves that can forestall the intraoperative identification of these nerves. The aim of this study is to provide a more pragmatic understanding of the variation of IHN, IIN, and GFN in relation to important surgical landmarks. While other studies have focused on the spinal nerve origins of the lumbar plexus (Klaassen et al., 2011) and nerve trajectory in the anterior abdominal wall, (Mandelkov and Loeweneck, 1988; Al-dabbagh, 2002; Whiteside et al., 2003; Ndiaye et al., 2007) this study focuses on branching patterns as found in the retroperitoneum. It should therefore aid clinicians performing retroperitoneal procedures, including laparoscopic triple neurectomies.

\section{MATERIALS AND METHODS}

A total of 22 cadavres were examined by dissection, including 10 formalin fixed cadavers and 12 fresh

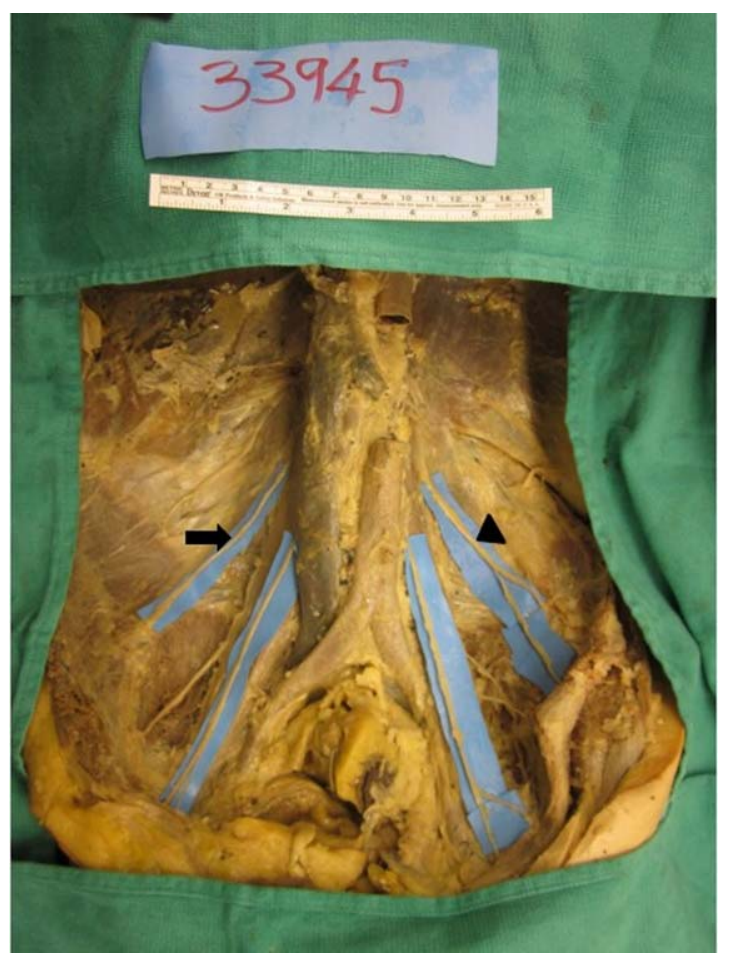

Fig. 1. Illustrates a dissected formalin fixed cadaver. The IHN-IIN bundle (superior blue bands) follows a type B branching pattern on the right (arrow) and a type $C$ on the left (arrowhead). Both GFN bundles (inferior blue bands) follow the classic type 1 pattern bilaterally. [Color figure can be viewed in the online issue, which is available at wileyonlinelibrary.com.]

specimens, generating 43 sets of data (GFN and IHN/ IIN were determined to be unusable in one specimen each). Demographic data consisting of age, gender, ethnicity, height, weight, BMI, and pertinent past medical and surgical history were recorded for each dissected body.

Dissections were performed by removing the anterior abdominal wall from the diaphragm superiorly to the pubic symphysis inferiorly. Abdominal structures including retroperitoneal organs were next removed to expose the posterior abdominal wall in the retroperitoneal space. The IHN and IIN were identified and isolated bilaterally using blunt dissection from point of emergence at the lateral border of the psoas major muscle to their respective points of entrance into the TA muscle. The GFN was similarly isolated from the point of emergence on the psoas major to approximately the level of the inguinal ligament (Fig. 1).

The following classifications and measurements regarding IHN, IIN, and GFN trajectories were documented:

Iliohypogastric and Ilioinguinal Nerves:

The pattern in which the IHN and IIN emerged from the lateral border of the psoas was categorized. Instances in which IHN and IIN emerged from the lateral psoas border as distinct branches were identified as type A. Type B was defined as specimens in which the IHN and IIN began as a common trunk and 
TABLE 1. Summary of Biometric Data

\begin{tabular}{|c|c|c|c|c|c|c|c|c|}
\hline ID & $\begin{array}{c}\text { State of } \\
\text { Preservation }\end{array}$ & Gender & Age & $\begin{array}{l}\text { Height } \\
(\mathrm{cm})\end{array}$ & Weight (kg) & BMI (kg/m2) & DA2A $(\mathrm{cm})^{\mathrm{a}}$ & DP2X $(\mathrm{cm})^{\mathrm{b}}$ \\
\hline $\begin{array}{l}33777 \\
34037 \\
34098 \\
33894 \\
5 \\
34222 \\
34107 \\
34102 \\
34100 \\
34223 \\
34224 \\
34234 \\
34231 \\
34230 \\
34233 \\
33668 \\
34232 \\
34238 \\
34229 \\
34101 \\
33921 \\
33945 \\
\text { Mean (SD) }\end{array}$ & $\begin{array}{l}\text { Preserved } \\
\text { Preserved } \\
\text { Preserved } \\
\text { Preserved } \\
\text { Fresh } \\
\text { Fresh } \\
\text { Preserved } \\
\text { Preserved } \\
\text { Preserved } \\
\text { Fresh } \\
\text { Fresh } \\
\text { Fresh } \\
\text { Fresh } \\
\text { Fresh } \\
\text { Fresh } \\
\text { Preserved } \\
\text { Fresh } \\
\text { Fresh } \\
\text { Fresh } \\
\text { Preserved } \\
\text { Fresh } \\
\text { Preserved }\end{array}$ & $\begin{array}{l}\text { Male } \\
\text { Male } \\
\text { Male } \\
\text { Female } \\
\text { Female } \\
\text { Female } \\
\text { Male } \\
\text { Female } \\
\text { Male } \\
\text { Female } \\
\text { Male } \\
\text { Female } \\
\text { Male } \\
\text { Male } \\
\text { Male } \\
\text { Female } \\
\text { Male } \\
\text { Female } \\
\text { Male } \\
\text { Female } \\
\text { Male } \\
\text { Male }\end{array}$ & $\begin{array}{c}88 \\
86 \\
62 \\
61 \\
31 \\
69 \\
98 \\
85 \\
87 \\
90 \\
81 \\
90 \\
80 \\
90 \\
96 \\
73 \\
69 \\
68 \\
63 \\
\end{array}$ & $\begin{array}{r}223.5 \\
218.4 \\
157.5 \\
154.9 \\
78.7 \\
175.3 \\
248.9 \\
215.9 \\
221.0 \\
228.6 \\
205.7 \\
228.6 \\
203.2 \\
228.6 \\
243.8 \\
185.4 \\
175.3 \\
172.7 \\
160.0\end{array}$ & $\begin{array}{r}79.3 \\
45.3 \\
63.4 \\
65.7 \\
75.7 \\
67.5 \\
43.9 \\
62.5 \\
49.8 \\
72.5 \\
56.6 \\
113.3 \\
76.6 \\
77.0 \\
42.6 \\
67.5 \\
113.3 \\
45.3 \\
86.1\end{array}$ & $\begin{array}{l}25.8 \\
18.3 \\
21.9 \\
27.4 \\
27.8 \\
22.7 \\
15.7 \\
21.6 \\
18.9 \\
21.1 \\
21.5 \\
32.1 \\
26.5 \\
24.4 \\
15.2 \\
22.0 \\
38.0 \\
12.5 \\
30.7\end{array}$ & $\begin{array}{r}21.0 \\
23.5 \\
23.3 \\
22.0 \\
23.5 \\
25.3 \\
23.0 \\
24.0 \\
24.3 \\
25.8 \\
23.0 \\
22.8 \\
23.3 \\
23.5 \\
25.5 \\
25.5 \\
24.5 \\
22.0 \\
24.3 \\
25.5 \\
25.5 \\
24.0 \\
23.9 \pm 1.3\end{array}$ & $\begin{array}{c}27.5 \\
33.0 \\
39.0 \\
33.0 \\
36.8 \\
37.3 \\
35.3 \\
34.0 \\
31.0 \\
34.5 \\
35.0 \\
39.0 \\
35.5 \\
30.0 \\
31.5 \\
28.8 \\
\\
37.5 \\
\\
37.0 \\
37.0 \\
39.5 \\
34.6 \pm 3.4\end{array}$ \\
\hline
\end{tabular}

a Distance from ASIS to ASIS.

bMidline distance from the pubic symphysis to the diaphragm.

proceeded to split before entering the TA. Type C denoted cases where the IHN and IIN emerged from the lateral psoas border and entered the TA as a common trunk. Regardless of the assigned category, it was then determined if any of these nerves emerged at the psoas-diaphragm junction (PDJ), defined as the intersection point of the medial crus of the diaphragm and the lateral edge of the psoas muscle. In the case where the nerves emerged distal to the PDJ, a linear distance along the lateral edge of the psoas muscle from the PDJ to the emergence point of the nerve was recorded. Additionally, in type A specimens, a series of measurements were taken from the emergence points of the IHN and IIN to their respective entrance points into the TA. The series of measurements consisted of a point-to-point measurement as well as horizontal ' $x$ ' and vertical ' $y$ ' distances in a coronal plane. In type $B$ specimens, a 3-component series of measurements, similar to that described for type A specimens, were made between the nerve split point and the transverse process of the $L 2$ vertebra. This point was located by palpating along the lateral edge of the spinal column inferiorly beginning at the T12 rib base until a major bony prominence was felt. The 3component series of measurements were then made between the nerve split point and the respective entries of the IHN and IIN into the TA. A 3-component series of measurements were conducted in type $C$ specimens from the point of nerve emergence at the lateral psoas border to the entrance site into the TA.

Genitofemoral Nerve:

GFN specimens were grouped into categories determined by the nature of nerve emergence on the anterior psoas major surface. Cases where the nerve emerged as a single trunk and proceeded to split into genital and FBs were deemed type 1 . Type 2 was characterized by a single GFN emerging from the psoas and coursing distally to the inguinal ligament with no splitting. Type 3 described cases in which the GFN emerged from the psoas as two or more branches. The third of the psoas muscle (proximal, middle, distal) where the GFN emerged was documented, for each category. Nerve emergence was defined as proximal if it occurred superior to the $\mathrm{L} 2$ transverse process; middle when it emerged between the L2 transverse process and iliac crest; and distal when it emerged inferior to the iliac crest. The distance between nerve emergence points and PDJ as well as the base of T12 rib was recorded for each specimen using the previously mentioned 3component series of measurements. The base of T12 rib was located by palpating the 12 th rib and moving medially along the inferior edge until the T12 vertebra was reached. Each point of nerve emergence from the psoas was then measured directly to the great vessel on the respective side being analyzed (the aorta on the left, the inferior vena cava on the right). Additionally in type A specimens, a linear distance from the point of emergence on the psoas to the point of the nerve split was recorded.

To ensure uniformity among data sets, all dissections and measurements were performed by the same two individuals. The measurements were taken independently by the two researchers to the nearest $0.5 \mathrm{~cm}$ using a tape measure and the mean of both measurements were recorded. Photographs of each specimen were taken.

To determine any statistically significant differences between measured variables, multiple statistical tests 
TABLE 2. Mean Distance of the GFN, GB, and FB From the Base of the T12 Rib and Mean Distance of the GB and FB From the Great Vessels

\begin{tabular}{llr}
\hline Variable (cm) & Gender & Mean (SD) \\
\hline $\begin{array}{l}\text { Distance from GFN } \\
\text { Bundle to the }\end{array}$ & Male & $1.7 \pm 1.5$ \\
$\begin{array}{l}\text { base of the T12 Rib [X] } \\
\text { Distance from GFN }\end{array}$ & Female & $-0.3 \pm 2.2$ \\
$\quad$ Bundle to the & Male & $7.6 \pm 2.4$ \\
$\quad$ base of the T12 Rib [Y] & Female & $9.5 \pm 2.3$ \\
Distance from & Male & $0.9 \pm 0.7$ \\
$\quad$ Great Vessels to FB & Female & $3.2 \pm 1.4$ \\
Distance from Great & Male & $0.5 \pm 0.5$ \\
Vessels to GB & Female & $2.3 \pm 1.0$ \\
\hline
\end{tabular}

were run; $P$-values less than 0.05 were considered significant. Fisher exact tests or $\chi^{2} \mathrm{~d}$ tests were performed to compare the various branching patterns of both nerve bundles to gender and to the side of body the nerves originated (left versus right). MANOVAs were performed to compare gender, side of body, and state of preservation, in turn, to each of the measurements. To ascertain statistically significant correlations, simple linear regression analyses were carried out using the various recorded measurements as dependent variables against the independent variables - Age, BMI, Height and Weight. SAS 9.4 and SPSS 22 were software programs used for the statistical analyses.

\section{RESULTS}

The independent variables and standardization measurements for the cadavers are summarized in Table 1. A statistically significant association was noted between GFN branching pattern and point of exit of the GFN bundle on the psoas $(P=0.032)$. No statistically significant differences were noted between Gender or Side and the branching pattern of the IHNIIN bundle $(P=0.32$ and $P=0.58$, respectively); similarly no statistically significant differences were noted between Gender or Side and the branching pattern of the GFN bundle ( $P=0.52$ and $P=1.00$, respectively). No statistically significant differences were noted between Gender or Side and point of exit of the GFN bundle on the psoas muscle $(P=0.78$ and $P=0.54$, respectively).

Results from the MANOVA showed significant differences only between Gender and the following variables:

Distance from the GFN Bundle to the Base of the T12 Rib X-component $(F=4.88, P=0.035)$

Distance from the GFN Bundle to the Base of the T12 Rib Y-component $(F=5.19, P=0.030)$

Distance from the Great Vessels to the GB of the GFN $(F=9.08, P=0.024)$

Distance from the Great Vessels to FB of the GFN $(F=11.94, P=0.014)$.

The means for these values by gender are summarized in Table 2. No statistically significant correlations were noted from simple linear regression models of the various measurements against age, BMI, height and weight.

\section{IHN and IIN Branching}

The type A branching pattern was the most common pattern accounting for $47 \%$ of the specimens. Type C accounted for $28 \%$ and this was closely matched by type B with $26 \%$. Figure 2 illustrates this data.

\section{IHN and IIN and Distances from Landmarks}

Table 3 illustrates the mean vertical distances and standard deviations of the IHN, IIN, and IHN-IIN

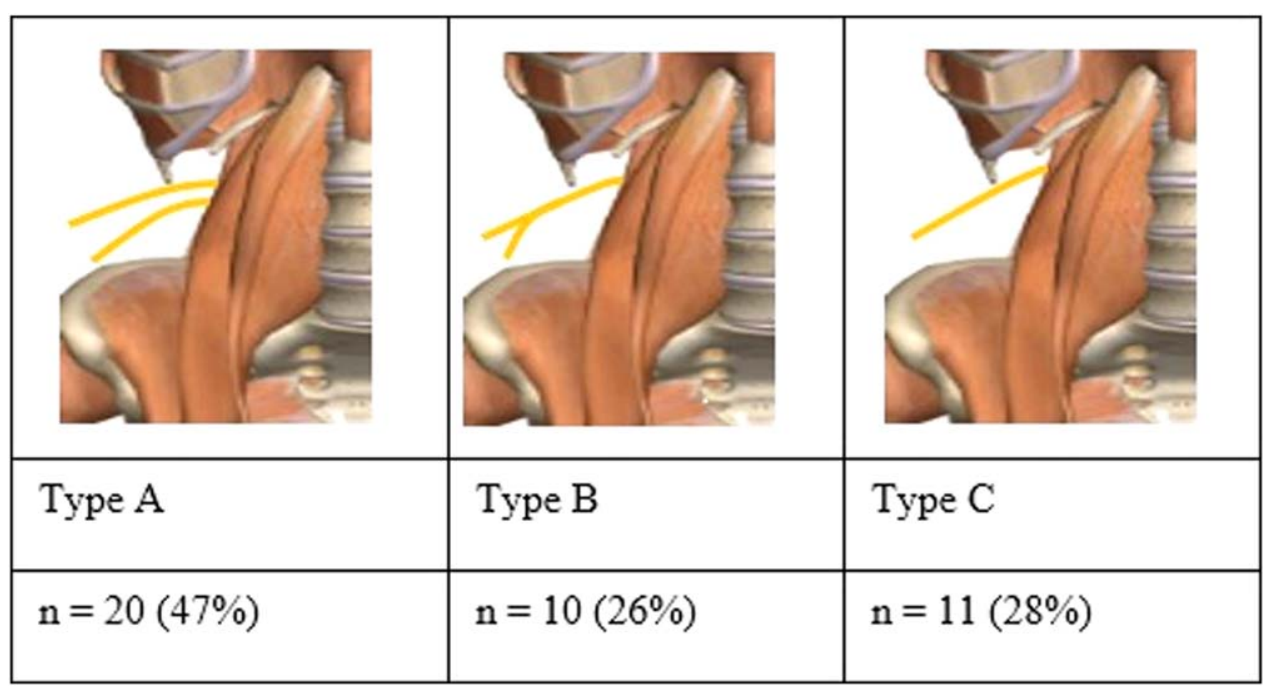

Fig. 2. IHN and IIN branching patterns and frequency of occurrence noted in study. [Color figure can be viewed in the online issue, which is available at wileyonlinelibrary.com.] 
TABLE 3. Mean Vertical Distances and Standard Deviations of the IHN, IIN, and IHN-IIN Bundle From the PDJ

\begin{tabular}{lc}
\hline IHN-IIN bundle type & $\begin{array}{c}\text { Mean distance }(\mathrm{cm}) \\
\text { from PDJ }\end{array}$ \\
\hline IHN-IIN Bundles (type B and C) & $2.7 \pm 1.5$ \\
IHN (type A) & $2.5 \pm 1.6$ \\
IIN (type A) & $4.7 \pm 2.4$ \\
\hline
\end{tabular}

bundle from the PDJ. The IHN-IIN bundle which constitutes of both types B and C (54\% of the observed specimens) had a mean distance of $2.7 \pm 1.5 \mathrm{~cm}$ from the PDJ.

The type A branching pattern produced two measurements for the IHN and IIN respectively. The IHN had a mean distance of $2.5 \pm 1.6 \mathrm{~cm}$ from the PDJ. The IIN was measured to be $4.7 \pm 2.4 \mathrm{~cm}$ from the PDJ.

\section{GFN branching and point of emergence on psoas major muscle}

The type 1 branching pattern was the most common branching pattern for the GFN accounting for $50 \%$ of the specimens. This was followed by the type $2(30 \%)$ and type $3(8 \%)$ branching patterns (Fig. 3). The GFN exited in $70 \%$ of the cases from the middle third of the psoas muscle, $20 \%$ from the distal third and $10 \%$ from the proximal third. Table 4 illustrates a cross-tabulation of GFN exit point against GFN branching pattern. Figure 4 shows a clustered bar-chart of the aforementioned variables.

\section{GFN and Mean Distances from Landmarks}

GFN to point of split. The type 1 branching pattern involved the GFN splitting into the genital and FBs after exiting the psoas muscle. The split was meas- ured at a mean vertical of distance of $8.6 \pm 3.5 \mathrm{~cm}$ from the point of emergence.

GFN to great vessels. Given the proximity of the GFN to the great vessels (aorta on the left side and inferior vena cava on the right) and the risk of damaging these vessels during a surgical procedure, the mean horizontal distance of the GFN from the great vessels was determined to be $1.8 \pm 1.7 \mathrm{~cm}$ in the type 1 and type 2 cases, while in the type 3 branching pattern the GB was located $1.2 \pm 1.2 \mathrm{~cm}$ and the $\mathrm{FB} 1.6 \pm 1.5 \mathrm{~cm}$ from these vessels, as illustrated in Table 5.

GFN and the base of the T12 rib. Table 5 illustrates the mean vertical and horizontal distances of the GFN types from the base of the T12 rib. In the case of the GFN bundle, the mean vertical and horizontal distances were determined to be $8.4 \pm 2.5 \mathrm{~cm}$ and $1.2 \pm 1.9 \mathrm{~cm}$, respectively. The GBs had mean distances of $11.8 \pm 3.1 \mathrm{~cm}$ in the vertical plane and $1.5 \pm 1.8 \mathrm{~cm}$ in the horizontal plane. The FBs had mean distances of $8.9 \pm 2.5 \mathrm{~cm}$ and $0.8 \pm 1.8 \mathrm{~cm}$ in the vertical and horizontal planes, respectively.

\section{DISCUSSION}

Postherniorrhaphy inguinodynia is a complication of inguinal hernia repair, a procedure performed approximately 20 million times annually, with approximately $8,00,000$ of those performed in the US alone (Chen et al., 2013). Laparoscopic triple neurectomy has proven effective, when conservative measures fail, in the management of the aforementioned complication, with $80 \%$ to $100 \%$ of patients reporting statistically significant improvement in pain levels post-operatively up until 3 years of follow-up (Song et al., 2011; Chen et al., 2013; Fischer, 2013; Mahan et al., 2014). While prior publications have commented on variations in the branching pattern of the IHN, IIN, and GFN, (Song et al., 2011; Chen et al., 2013; Tender and Serban,

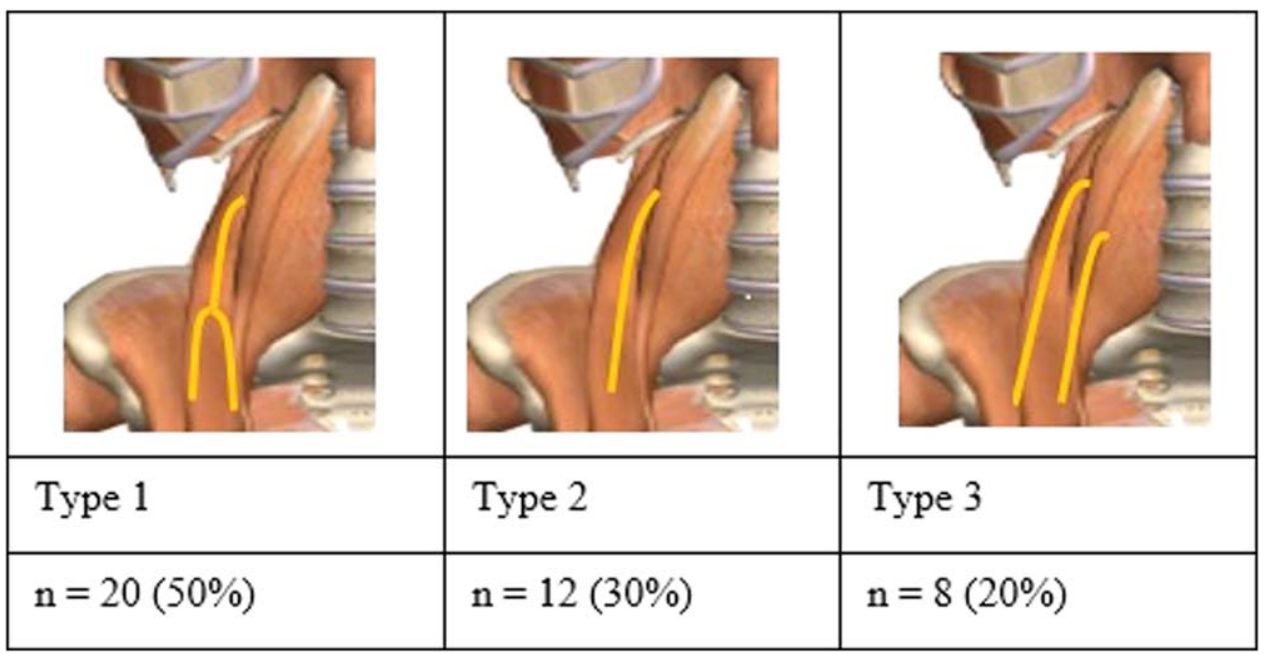

Fig. 3. GFN branching patterns and frequency of occurrence noted in study. [Color figure can be viewed in the online issue, which is available at wileyonlinelibrary.com.] 
TABLE 4. Cross-tabulation of GFN exit point against GFN branching pattern

\begin{tabular}{lcccc}
\hline & \multicolumn{4}{c}{$\begin{array}{c}\text { GFN branching pattern } \\
\text { Frequency }(n)\end{array}$} \\
\cline { 2 - 5 } & \multicolumn{4}{c}{ Percent (\%) } \\
\cline { 2 - 5 } GFN Exit Point & Type 1 & Type 2 & Type 3 & Total \\
\hline Proximal & 3 & 0 & 1 & 4 \\
Middle & 7.5 & 0 & 2.5 & 10 \\
& 16 & 9 & 3 & 28 \\
Distal & 40 & 22.5 & 7.5 & 70 \\
Total & 1 & 3 & 4 & 8 \\
& 2.5 & 7.5 & 10 & 20 \\
& 20 & 12 & 8 & 40 \\
& 50 & 30 & 20 & 100 \\
\hline
\end{tabular}

2013) none so far have provided a characterization of these variations, especially in the retroperitoneum where the neurectomies are performed. This study provides a topographic map of the IHN, IIN, and GFN in the retroperitoneum with respect to various anatomical landmarks which can be observed during laparoscopy.

On dissection, the IHN-IIN bundle followed three distinct patterns. The classic anatomical description (type A) has the IHN and IIN exiting behind the psoas major as separate nerves; this was observed in $47 \%$ of the specimens. However, in $53 \%$ of the specimens, the nerves exited as a single bundle and either split (type B [26\%]) or not (type C [28\%]). The PDJ was used as a point of reference to gauge the distance to the various nerves as illustrated in Table 3. As noted, there was significant variation in branching pattern, however, this difference did not correlate in a statistically significant way with either gender or side of the body. There were no statistically significant correlations between the various IHN-IIN measurements and the independent variables.

On dissection, the GFN bundle followed three distinct patterns. The classical description labeled type 1 where the GFN exits as a single nerve on the psoas and splits into the genital and FBs was noted in only $44 \%$ of the specimens; in $56 \%$ of the specimens the GFN exited and did not split (type 2 [32\%]) or exited as separate branches (type 3 [24\%]). Tender et al. describe a lateral retroperitoneal transpsoas approach to lumbar fusion (2013). This involves dissection of the psoas, which necessitates an understanding of the variation of the GFN bundle and its exit point which is crucial to procedures involving this muscle. The GFN was further characterized based upon what third of

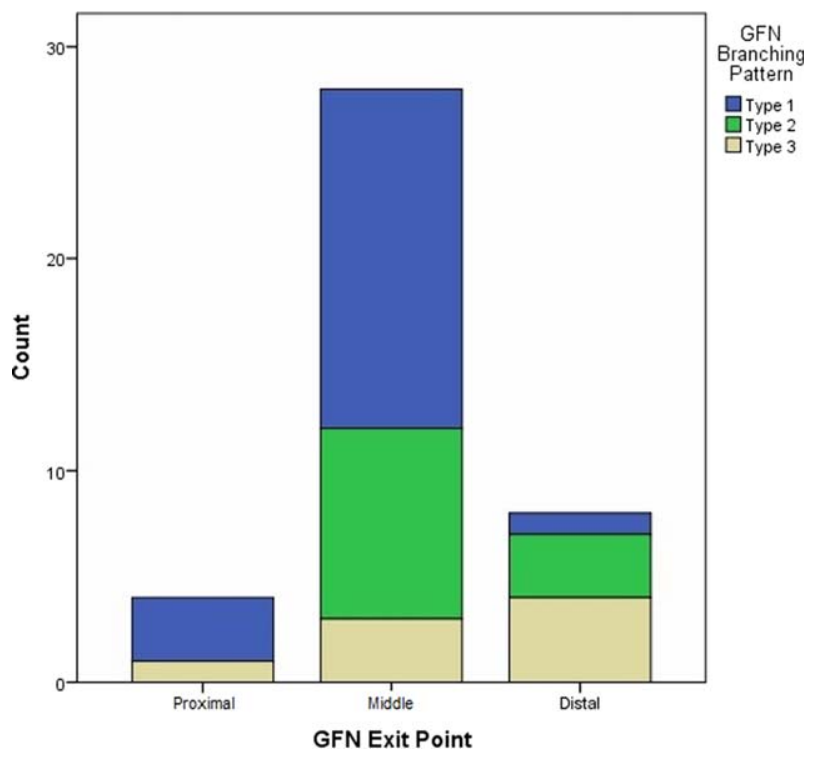

Fig. 4. Chart illustrating the frequencies of the point of emergence of the GFN on the psoas major muscle delineated by branch patterns. [Color figure can be viewed in the online issue, which is available at wileyonlinelibrary.com.]

the psoas it exited; the vast majority exited on the middle third $(70 \%), 20 \%$ from the distal third, and $10 \%$ from the proximal third. On considering the branch patterns the aforementioned distribution held for types 1 and 2 but not for type 3 as illustrated in Table 4. Per the Fisher exact test this difference is statistically significant.

In order to provide dimensionality to the map, the mean horizontal distance to the great vessels of the GFN bundle, the mean vertical and horizontal distance to the base of the T12 rib were obtained. Per the results, in the type $\mathrm{C}$ branching pattern, the $\mathrm{FB}$ and the GB ran closer to the great vessels in males than females. The mean distance from the base of the T12 rib to the GFN bundle was noted to be more lateral and superior compared to females. Though these differences in measurements are statistically significant, they may not necessarily be clinically relevant. There were no statistically significant correlations between the various GFN measurements and the independent variables (age, weight, height, BMI).

There is always the possibility that the measurements could be underestimated (due to rigidity of fixed specimens) or exaggerations (due to sagging in

TABLE 5. Mean distances of the GFN bundle, GB and FB from their point of emergence to the Great vessels, Split Point as applicable, and base of the T12 rib (horizontal and vertical components)

\begin{tabular}{lcccc}
\hline & $\begin{array}{c}\text { Mean distance from } \\
\text { point of emergence } \\
\text { to great vessels } \\
(\mathrm{cm})\end{array}$ & $\begin{array}{c}\text { Mean distance } \\
\text { from point of } \\
\text { emergence to split } \\
\text { point (cm) }\end{array}$ & $\begin{array}{c}\text { Mean distance from } \\
\text { base of T12 to point of } \\
\text { emergence (cm) } \\
\text { X-component }\end{array}$ & $\begin{array}{c}\text { Mean distance from } \\
\text { base of T12 to point of } \\
\text { emergence (cm) } \\
\text { y-component }\end{array}$ \\
\hline GFN bundle (type 1 and 2) & $1.8 \pm 1.7$ & $8.6 \pm 3.5$ & $1.2 \pm 1.9$ & $8.4 \pm 2.5$ \\
GB (type 3) & $1.2 \pm 1.2$ & N/A & $1.5 \pm 1.8$ & $11.8 \pm 3.1$ \\
FB (type 3) & $1.6 \pm 1.5$ & N/A & $0.8 \pm 1.8$ & $8.9 \pm 2.5$ \\
\hline
\end{tabular}


fresh specimens). However, from the MANOVA findings, no statistically significant differences were noted between the state of preservation and the various measurements. The linear regression models showed no statistically significant correlations. Nevertheless, the study did achieve the original goal of characterizing the branching patterns of the nerve bundles in the retroperitoneal region.

\section{ACKNOWLEDGMENTS}

The authors are thankful and appreciative to all the anatomical donors and their families and also for the help provided by Dean Mueller (Director of the UMMS Anatomical Donations Program), in the procurement of specimens and for providing the relevant biometric information.

\section{REFERENCES}

Al-dabbagh AKR. 2002. Anatomical variations of the inguinal nerves and risks of injury in 110 hernia repairs. Surg Radiol Anat 24: 102-107.

Amid PK. 2004. Causes, prevention, and surgical treatment of postherniorrhaphy neuropathic inguinodynia: Triple neurectomy with proximal end implantation. Hernia 8:343-349.

Arslan OE. 2005. Anatomy of the abdominal wall. In: Shiffman MA Mirafati S, editors. Aesthetic Surgery of the Abdominal Wall. Springer-Verlag. p 1-28.

Chen DC, Hiatt JR, Amid PK. 2013. Operative management of refractory neuropathic inguinodynia by a laparoscopic retroperitoneal approach. JAMA Surg 148:962-967.

Clemente CD. 1997. Section III: The abdomen. In: Anatomy: A Regional Atlas of the Human Body, 4th Ed. Baltimore, MD: Lippincott Williams and Wilkins. p 238.

Courtney CA, Duffy K, Serpell MG, O'Dwyer PJ. 2002. Outcome of patients with severe chronic pain following repair of the groin hernia. Br J Surg 89:1310-1314.

Cunningham J, Temple WJ, Mitchell P, Nixon JA, Preshaw RM, Hagan NA. 1996. Cooperative hernia study: Pain in the post-repair patient. Ann Surg 224:598-602.
Fischer JE. 2013. Hernia repair: Why do we continue to perform mesh repair in the face of the human toll of inguinodynia?. Am J Surg 206:619-623.

Giger U, Wente MN, Buchler MW, Krahenbuhl S, Lerut J, Krahenbuhl L. 2009. Endoscopic retroperitoneal neurectomy for chronic pain after groin surgery. Br J Surg 96:1076-1081.

Klaassen Z, Marshall E, Tubbs RS, Louis RG, Jr. Warrtmann CT, Loukas M. 2011. Anatomy of the ilioinguinal and iliohypogastric nerves with observations of their spinal nerve contributions. Clin Anat 24:454-461.

Krahenbuhl L, Striffeler H, Baer HU, Buchler MW. 1997. Retroperitoneal endoscopic neurectomy for nerve entrapment after hernia repair. Br J Surg 84:216-219.

Mahan MA, Kader AK, Brown JM. 2014. Robot-assisted triple neurectomy for iatrogenic inguinal pain: A technical note. Acta Neurochirurgica 156:171-175.

Mandelkow H, Loeweneck H. 1988. The iliohypogastric and ilioinguinal nerves: Distribution in the abdominal wall, danger areas in surgical incisions in the inguinal and pubic regions and reflected visceral pain in their dermatomes. Surg Radiol Anat $10: 145-149$.

Moore KL, Dalley AF. 2006. Ch. 2: Abdomen. In: Clinically Oriented Anatomy. 5th Ed. Baltimore, MD: Lippincott Williams and Wilkins. p 336.

Muto CM, Pedana N, Scarpelli S, Galardo R, Guida G, Schiavone V. 2005. Inguinal neurectomy for nerve entrapment after open/laparoscopic hernia repair using retroperitoneal endoscopic approach. Surg Endosc 19:974-976.

Ndiaye A, Diop M, Ndoye M, Konate I, Ndiaye, AI, Mane L et al 2007. Anatomical basis of the neuropathies and damage of the ilioinguinal nerve during repairs of groin hernias. Surg Radiol Anat 29:675-681.

Netter FH. 2006. Section IV: Abdomen. In: Atlas of Human Anatomy. 4th Ed. Philadelphia, PA: Saunders Elsevier. p 267.

Song JW, Wolf JS, McGillicuddy JE, Bhangoo S, Yang LJS. 2011. Laparoscopic triple neurectomy for intractable groin pain: Technical report of 3 cases. Operat Neurosurg 68:339-346.

Tender GC, Serban D. 2013. Genitofemoral nerve protection during the lateral retroperitoneal transpsoas approach. Neurosurgery 73:192-196. discussion 196-7.

Whiteside JL, Barber MD, Walters MD, Falcone T. 2003. Anatomy of the ilioinguinal and iliohypogastric nerves in relation to trocar placement and low transverse incisions. Am J Obstet Gynecol 189:1574-1578. 